\title{
Even Dimensional Generalization of Chern-Simons Action and New Gauge Symmetry
}

\author{
Noboru Kawamoto and Yoshiyuki Watabiki \\ Department of Physics, Kyoto University, Kyoto 606, Japan
}

Received June 6, 1991

\begin{abstract}
We propose a new even-dimensional action which shares close algebraic similarities with the Chern-Simons action and thus possesses a topological nature. This action has a new type of gauge symmetry in the sense that adjoint representation is not enough to close the gauge transformation and gauge fermions are incorporated. "Quaternionic structure" emerges as a natural algebra to control the different natures of even forms, odd forms, bosons and fermions. We claim that the bundle structure in consideration is mathematically a new object.
\end{abstract}

Recently three-dimensional Chern-Simons action [1] played a fundamental role to understand two-dimensional conformal field theory [2] and three-dimensional gravity [3]. It has, however, been well known that Chern-Simons action is applicable only in three dimensions.

In this paper we show that it is possible to construct a new even-dimensional action which shares close algebraic similarities with the Chern-Simons action and possesses a new type of gauge symmetry. We claim that this construction defines mathematically a new "bundle structure" and topological invariants. It is obviously impossible to extend naively the three-dimensional Chern-Simons action into even dimensions. We need to introduce new ingredients and structures. In this paper we introduce antisymmetric tensors, gauge fermions and "quaternionic structure." There have been several trials of topological field theories in connection with Chern-Simons action and anti symmetric tensors $[4,5]$.

We first recall the procedure to prove the gauge invariance of the standard three-dimensional Chern-Simons action

$$
S=\int \operatorname{Tr}\left(\frac{1}{2} \operatorname{Ad} A+\frac{1}{3} A^{3}\right),
$$

where $A$ is one form gauge field and carries non-Abelian gauge suffix. The above action is invariant under the following gauge transformation: $\delta A=d v+[A, v]$, where $v$ is a zero form gauge parameter. We need the following two fundamental properties to prove the gauge invariance of the action: 
A) Cyclic invariance of elements in the trace,

B) $\{\vec{d}, A\}=d A,[\vec{d}, v]=d v$.

The arrow over the exterior derivative operator denotes a differential operation on any fields on the right of the arrow. We investigate how we can generalize the above two properties A) and B).

We first introduce the new definitions of a gauge field $\mathscr{A}$ and an exterior derivative operator $Q$ and a gauge parameter $\mathscr{V}$ in the following:

$$
\begin{aligned}
& \mathscr{A}=\psi \mathbf{1}+\hat{\psi} \mathbf{i}+A \mathbf{j}+\hat{A} \mathbf{k}, \\
& Q=\mathbf{j} d, \\
& \mathscr{V}=\hat{a} \mathbf{1}+a \mathbf{i}+\hat{\alpha} \mathbf{j}+\alpha \mathbf{k},
\end{aligned}
$$

where $d=d x^{\mu} \partial_{\mu}$. Here we have classified gauge fields and parameters by the following notations: Roman and Greek characters are bosonic and fermionic fields or parameters respectively and the hat denotes even forms in contrast with non-hat odd forms. The different natures of even form, odd form, fermion and boson are classified by $\mathbf{1}, \mathbf{i}, \mathbf{j}, \mathbf{k}$ carrying "quaternionic structure" which will be defined more precisely later. To be explicit the gauge fields; $\hat{A}$ (bosonic even form), $A$ (bosonic odd form), $\hat{\psi}$ (fermionic even form), and $\psi$ (fermionic odd form) can be expressed as

$$
\begin{aligned}
& \hat{A}=\frac{T_{a}}{2}\left(A^{(0) a}+\frac{1}{2} A_{\mu \nu}^{(2) a} d x^{\mu} \wedge d x^{\nu}+\ldots\right), \\
& A=\frac{T_{a}}{2}\left(A_{\mu}^{(1) a} d x^{\mu}+\frac{1}{3 !} A_{\mu \nu \varrho}^{(3) a} d x^{\mu} \wedge d x^{\nu} \wedge d x^{\varrho}+\ldots\right), \\
& \hat{\psi}=\frac{T_{a}}{2}\left(\psi^{(0) a}+\frac{1}{2} \psi_{\mu \nu}^{(2) a} d x^{\mu} \wedge d x^{\nu}+\ldots\right), \\
& \psi=\frac{T_{a}}{2}\left(\psi_{\mu}^{(1) a} d x^{\mu}+\frac{1}{3 !} \psi_{\mu \nu \varrho}^{(3) a} d x^{\mu} \wedge d x^{\nu} \wedge d x^{\varrho}+\ldots\right),
\end{aligned}
$$

where $A^{(p) a}$ denotes a bosonic $p$-form function while $\psi^{(p) a}$ denotes a fermionic $p$-form function and $T_{a}$ is a generator of a certain gauge algebra. The degree of the highest form of $\hat{A}$ or $A$, and $\hat{\psi}$ or $\psi$ coincides with the dimension of the space time. The gauge parameters $\hat{a}, a, \hat{\alpha}$, and $\alpha$ have the similar forms as the gauge fields. As a base manifold we consider an $N$-dimensional compact manifold without boundary.

Any gauge fields or parameters can be written in a form; $\lambda=l_{\mathbf{1}} \mathbf{1}+l_{\boldsymbol{i}} \mathbf{i}+l_{\mathbf{j}} \mathbf{j}+l_{\boldsymbol{k}} \mathbf{k}$. We classify them into two categories: $\lambda \in \Lambda_{1}$ if $\left(l_{1}, l_{i}, l_{j}, l_{k}\right)=($ bosonic even form, bosonic odd form, fermionic even form, fermionic odd form) and $\lambda \in \Lambda_{k}$ if $\left(l_{1}, l_{i}, l_{j}, l_{k}\right)$ $=$ (fermionic odd form, fermionic even form, bosonic odd form, bosonic even form). It is clear that $\mathscr{A}, Q \in \Lambda_{k}$ and $\mathscr{V} \in \Lambda_{1}$. In order to get closed algebra under a multiplication we need the following relations:

$$
\begin{aligned}
\mathbf{1}^{2} & =\mathbf{i}^{2}=\mathbf{j}^{2}=\mathbf{k}^{2}= \pm \mathbf{1}, \\
\mathbf{j} \mathbf{j} & = \pm \mathbf{j i}= \pm \mathbf{k} \\
\mathbf{j} \mathbf{k} & = \pm \mathbf{k j}= \pm \mathbf{i} \\
\mathbf{k} \mathbf{i} & = \pm \mathbf{i k}= \pm \mathbf{j}
\end{aligned}
$$


where signs are irrelevant here. It is then easy to show that

$$
\Lambda_{1} \Lambda_{1} \sim \Lambda_{1}, \quad \Lambda_{1} \Lambda_{k} \sim \Lambda_{k}, \quad \Lambda_{k} \Lambda_{k} \sim \Lambda_{1},
$$

where the first equation means the following: if $\lambda_{1}, \lambda_{1}^{\prime} \in \Lambda_{1}$ then $\lambda_{1} \lambda_{1}^{\prime} \in \Lambda_{1}$ and similar for the other two equations.

We can now show

$$
\operatorname{Tr}_{\mathbf{1}}\left(\lambda_{k} \lambda_{1}\right)=\operatorname{Tr}_{\mathbf{1}}\left(\lambda_{1} \lambda_{k}\right), \quad \operatorname{Tr}_{\mathbf{k}}\left(\lambda_{k} \lambda_{1}\right)=\operatorname{Tr}_{\mathbf{k}}\left(\lambda_{1} \lambda_{k}\right),
$$

where $\lambda_{1} \in \Lambda_{1}, \lambda_{k} \in \Lambda_{k}$ and if the following relations are satisfied

$$
\mathbf{i j}=-\mathbf{j i}, \quad \mathbf{j k}=-\mathbf{k j},
$$

besides the relations (4). In the $\operatorname{Tr}_{1}$ we pick up the 1-th component and take the trace of the gauge algebra. It is similar for $\operatorname{Tr}_{\mathbf{k}}$. As far as 1-th and $\mathbf{k}$-th components are concerned the generalized version of the condition A) is obtained.

We can then show that the condition B) is satisfied in the general case:

$$
\begin{aligned}
& \{\vec{Q}, \mathscr{A}\}=Q \mathscr{A}, \\
& {[\vec{Q}, \mathscr{V}]=Q \mathscr{V},}
\end{aligned}
$$

if the relations (4) and (7) are satisfied.

We have thus obtained the generalized versions of the conditions A) and B) in (6) and (8). The relations (4) and (7) are necessary to prove both of them and lead to the constraints

$$
\begin{aligned}
& \mathbf{1}^{2}=\mathbf{1}, \quad \mathbf{i}^{2}=\varepsilon_{1} \mathbf{1}, \quad \mathbf{j}^{2}=\varepsilon_{2} \mathbf{1}, \quad \mathbf{k}^{2}=-\varepsilon_{1} \varepsilon_{2} \mathbf{1}, \\
& \mathbf{i j}=\mathbf{k}, \quad \mathbf{j k}=-\mathbf{k} \mathbf{j}=-\varepsilon_{2} \mathbf{i}, \quad \mathbf{k i}=-\mathbf{i k}=-\varepsilon_{1} \mathbf{j},
\end{aligned}
$$

where $\left(\varepsilon_{1}, \varepsilon_{2}\right)=(-1,-1),(-1,+1),(+1,-1),(+1,+1)$. In the case $\left(\varepsilon_{1}, \varepsilon_{2}\right)$ $=(-1,-1), \mathbf{1}, \mathbf{i}, \mathbf{j}, \mathbf{k}$ satisfy the algebra of quaternion. For the rest of the three cases $1, \mathbf{i}, \mathbf{j}, \mathbf{k}$ satisfy $g l(2, \mathbf{R})$ Lie algebra. We symbolically call these four different algebras as "quaternion algebra" and thus the generalized gauge fields and parameters carry "quaternionic structure."

We now define the following generalized Chern-Simons action:

$$
\begin{aligned}
& S_{\mathrm{e}}^{\mathrm{b}}=\int \operatorname{Tr}_{\mathbf{k}}\left(\frac{1}{2} \mathscr{A} Q \mathscr{A}+\frac{1}{3} \mathscr{A}^{3}\right), \\
& S_{0}^{\mathrm{f}}=\int \operatorname{Tr}_{\mathbf{1}}\left(\frac{1}{2} \mathscr{A} Q \mathscr{A}+\frac{1}{3} \mathscr{A}^{3}\right) .
\end{aligned}
$$

It should be noted that the Chern-Simons form in the trace belongs to $\Lambda_{k}^{3} \sim \Lambda_{k} \Lambda_{1} \sim \Lambda_{k}$ and thus $\mathrm{k}$-th component is a bosonic even form and 1-th component is a fermionic odd form.

By using the relations in Eqs. (6) and (8) we can prove that the above generalized Chern-Simons actions are invariant under the gauge transformation

$$
\delta \mathscr{A}=[\mathscr{D}, \mathscr{V}]=Q \mathscr{V}+[\mathscr{A}, \mathscr{V}]
$$

where $\mathscr{D}=\vec{Q}+\mathscr{A}$.

Introducing the defining relations of (2) into equations in (10), we obtain explicit forms of the actions

$$
\begin{aligned}
& S_{\mathrm{e}}^{\mathrm{b}}=\int \operatorname{Tr}\left\{\varepsilon_{2} \hat{A}\left(d A+A^{2}+\varepsilon_{1} \varepsilon_{2} \hat{\psi}^{2}+\varepsilon_{2} \psi^{2}\right)-\frac{1}{3} \varepsilon_{1} \varepsilon_{2} \hat{A}^{3}-\psi(d \hat{\psi}+[A, \hat{\psi}])\right\}, \\
& S_{0}^{\mathrm{f}}=\int \operatorname{Tr}\left\{\varepsilon_{2} \psi\left(d A+A^{2}+\varepsilon_{1} \varepsilon_{2} \hat{\psi}^{2}-\varepsilon_{1} \hat{A}^{2}\right)+\frac{1}{3} \psi^{3}+\varepsilon_{1} \varepsilon_{2} \hat{A}(d \hat{\psi}+[A, \hat{\psi}])\right\},
\end{aligned}
$$


which are invariant up to surface terms under the following gauge transformation:

$$
\begin{aligned}
& \delta A=d \hat{a}+[A, \hat{a}]+\varepsilon_{1}\{\hat{\psi}, \alpha\}+[\psi, \hat{\alpha}]-\varepsilon_{1}\{\hat{A}, a\}, \\
& \delta \hat{\psi}=-\varepsilon_{2} d \alpha-\varepsilon_{2}\{A, \alpha\}+[\hat{\psi}, \hat{a}]+[\psi, a]+\varepsilon_{2}\{\hat{A}, \hat{\alpha}\}, \\
& \delta \psi=\varepsilon_{2} d \hat{\alpha}+\varepsilon_{2}[A, \hat{\alpha}]+\varepsilon_{1}[\hat{\psi}, a]+[\psi, \hat{a}]-\varepsilon_{1} \varepsilon_{2}[\hat{A}, \alpha], \\
& \delta \hat{A}=-d a-\{A, a\}+\{\hat{\psi}, \hat{\alpha}\}+[\psi, \alpha]+[\hat{A}, \hat{a}],
\end{aligned}
$$

where $[$,$] and \{$,$\} are commutator and anti-commutator, respectively.$

It should be noted that $S_{\mathrm{e}}^{\mathrm{b}}$ is even-dimensional bosonic action while $S_{0}^{\mathrm{f}}$ is odddimensional fermionic one. The gauge transformation in (13) includes fermions as gauge fields and parameters. The transformation properties are, however, different from the standard supersymmetric one. We may call the fermions $\hat{\psi}$ and $\psi$ as "gauge fermions."

Here is one important point to be noted. The terms like $[\psi, \hat{\alpha}],\{\hat{A}, a\},[\psi, a]$, and $\{\hat{A}, \hat{\alpha}\}$ in the gauge transformation (13) have an unusual nature. They make it impossible to close the gauge algebra within the adjoint representation of a Lie algebra. We need an algebra which is closed by commutators and anticommutators. This is equivalent to say that a product of any two generators should belong to the generators again. The only possible generators satisfying these requirements consist of $n \times n$ matrices with elements that are either real, complex or quaternionic [6]. A specific example of the algebra is realized by Clifford algebra $[7,8]$. The corresponding gauge symmetry of the generalized Chern-Simons action is thus different from the standard gauge symmetry and may be identified as a new type of gauge symmetry.

We can now obtain the even-dimensional generalization of the standard threedimensional Chern-Simons action by taking pure bosonic sector of $S_{\mathrm{e}}^{\mathrm{b}}$ by putting fermion components zero:

$$
S_{\mathrm{e}}^{\mathrm{b}}=\int \operatorname{Tr} \varepsilon_{2}\left\{\widehat{A}\left(d A+A^{2}\right)-\frac{1}{3} \varepsilon_{1} \widehat{A}^{3}\right\},
$$

which is invariant under a gauge transformation

$$
\begin{aligned}
& \delta A=d \hat{a}+[A, \hat{a}]-\varepsilon_{1}\{\hat{A}, a\}, \\
& \delta \hat{A}=-d a-\{A, a\}+[\hat{A}, \hat{a}] .
\end{aligned}
$$

In the integrand of Eq. (14) we pick up the forms whose degree coincides with the dimension $N$. In the gauge transformation (15) the same order of the forms should be equated.

We can now write down very explicit expressions of the pure bosonic evendimensional actions. In two dimensions we define gauge fields and parameters as: $A=\omega, \hat{A}=\varepsilon_{2}\left(\phi+\varepsilon_{1} B\right), \hat{a}=v+\varepsilon_{1} b$, and $a=-\varepsilon_{1} \varepsilon_{2} u$, where $\phi, \omega$, and $B$ are zero, one, and two form gauge fields while $v, u$, and $b$ are zero, one, and two form gauge parameters, respectively. Substituting these definitions into Eqs. (14) and (15), we obtain the two-dimensional generalized Chern-Simons action

$$
S_{2}=\int \operatorname{Tr}\left\{\phi\left(d \omega+\omega^{2}\right)-\phi^{2} B\right\},
$$

which is invariant under the following gauge transformation:

$$
\begin{aligned}
& \delta \phi=[\phi, v], \\
& \delta \omega=d v+[\omega, v]+\{\phi, u\}, \\
& \delta B=d u+\{\omega, u\}+[B, v]+[\phi, b] .
\end{aligned}
$$


In four dimensions we define gauge fields and parameters as $A=\omega+\varepsilon_{1} \Omega$, $\hat{A}=\varepsilon_{2}\left(\varepsilon_{1} \phi+B+\varepsilon_{1} H\right), \hat{a}=v+\varepsilon_{1} b+V$, and $a=-\varepsilon_{2}\left(u+\varepsilon_{1} U\right)$, where $\phi, \omega, B, \Omega$, and $H$ are zero, one, two, three, and four form gauge fields while $v, u, b, U$, and $V$ are zero, one, two, three, and four form gauge parameters, respectively. By substituting these explicit expression into (14) and (15), we obtain the explicit form of the fourdimensional generalized Chern-Simons action

$$
S_{4}=\int \operatorname{Tr}\left\{B\left(d \omega+\omega^{2}\right)+\phi(d \Omega+\omega \Omega+\Omega \omega)-\phi B^{2}-\phi^{2} H\right\},
$$

which is invariant under the following gauge transformation

$$
\begin{aligned}
& \delta \phi=[\phi, v], \\
& \delta \omega=d v+[\omega, v]+\{\phi, u\}, \\
& \delta B=d u+\{\omega, u\}+[B, v]+[\phi, b], \\
& \delta \Omega=d b+[\omega, b]+[\Omega, v]+\{B, u\}+\{\phi, U\}, \\
& \delta H=d U+\{\omega, U\}+\{\Omega, u\}+[H, v]+[B, b]+[\phi, V] .
\end{aligned}
$$

Higher even-dimensional actions and the corresponding gauge transformation can be obtained in a similar way. Here we further need to specify the gauge algebra which should be closed within commutators and anti-commutators and can be realized by Clifford algebra. We carry out explicit analyses of the two-dimensional action (16) and the four-dimensional action (18) with the Clifford algebra in our separate papers, which provide two-dimensional gravity and four-dimensional gravity $[7,8]$.

It is interesting to note that the original actions in (10) have the same ChernSimons form while the explicit expressions of the pure bosonic actions have different forms depending on the dimensions. This double structure of the evendimensional action can be realized owing to the introduction of even forms, odd forms, gauge bosons and fermions through the quaternionic structure.

We now point out that the algebraic structure of the generalized gauge field is homomorphic to that of the standard gauge theory. The curvature of the generalized gauge field is given by

$$
\mathscr{F}=\mathscr{D}^{2}=Q \mathscr{A}+\mathscr{A}^{2},
$$

and the equation of motion of the generalized Chern-Simons action is

$$
\mathscr{F}=0 \text {, }
$$

which is locally flat condition of the "bundle" in consideration. forms

Pure bosonic version of the equations of motion in terms of $A$ and $\hat{A}$ have the

$$
\begin{aligned}
d A+A^{2}-\varepsilon_{1} \hat{A}^{2} & =0, \\
d \hat{A}+[A, \widehat{A}] & =0,
\end{aligned}
$$

which are obtained by the vanishing conditions of the coefficients for $\mathbf{1}$ and $\mathbf{i}$ of the curvature $\mathscr{F}$.

Explicit forms of the equations of motion in two dimensions and four dimensions can be obtained substituting the defining equations of $A$ and $\hat{A}$ into Eqs. (22). The equations of motion in two dimensions corresponding to the action 
$S_{2}$ are

$$
\begin{aligned}
\phi^{2} & =0, \\
d \phi+[\omega, \phi] & =0, \\
d \omega+\omega^{2}-\{\phi, B\} & =0 .
\end{aligned}
$$

The equations of motions of the four-dimensional generalized action $S_{4}$ are

$$
\begin{aligned}
\phi^{2} & =0, \\
d \phi+[\omega, \phi] & =0, \\
d \omega+\omega^{2}-\{\phi, B\} & =0, \\
d B+[\omega, B]+[\Omega, \phi] & =0, \\
d \Omega+\{\omega, \Omega\}-B^{2}-\{\phi, H\} & =0 .
\end{aligned}
$$

We can now show that the following familiar relations hold in the generalized case even with fermions:

$$
\begin{aligned}
\operatorname{Tr}_{\mathbf{i}}\left\{\mathscr{F}^{2}\right\} & =\operatorname{Tr}_{\mathbf{i}}\left\{Q\left(\mathscr{A} Q \mathscr{A}+\frac{2}{3} \mathscr{A}^{3}\right)\right\}, \\
\operatorname{Tr}_{\mathbf{j}}\left\{\mathscr{F}^{2}\right\} & =\operatorname{Tr}_{\mathbf{j}}\left\{Q\left(\mathscr{A} Q \mathscr{A}+\frac{2}{3} \mathscr{A}^{3}\right)\right\},
\end{aligned}
$$

where $\mathbf{i}$-sector of the trace is valid in odd dimensions while $\mathbf{j}$-sector is valid in even dimensions. It should be noted that we have to take now $\mathbf{i}$ - or $\mathbf{j}$-sector of the trace because the gauge invariant sectors within the trace have turned into $i-$ and j-sectors because of the additional $Q$ insertion to the generalized Chern-Simons action. In other words the following relations are necessary to prove the relations (25):

$$
\operatorname{Tr}_{\mathbf{i}}\left(\lambda_{1} \lambda_{1}^{\prime}\right)=\operatorname{Tr}_{\mathbf{i}}\left(\lambda_{1}^{\prime} \lambda_{1}\right), \quad \operatorname{Tr}_{\mathbf{j}}\left(\lambda_{1} \lambda_{1}^{\prime}\right)=\operatorname{Tr}_{\mathbf{j}}\left(\lambda_{1}^{\prime} \lambda_{1}\right),
$$

where $\lambda_{1}, \lambda_{1}^{\prime} \in \Lambda_{1}$ while it should be noted that $\mathscr{F}, Q \mathscr{A}, \mathscr{A}^{2} \in \Lambda_{1}$. In general we obtain

$$
\begin{aligned}
\operatorname{Tr}_{\mathbf{i}}\left\{\mathscr{F}^{n}\right\} & =\operatorname{Tr}_{\mathbf{i}}\left\{Q \Omega_{2 n-1}\right\}, \\
\operatorname{Tr}_{\mathbf{j}}\left\{\mathscr{F}^{n}\right\} & =\operatorname{Tr}_{\mathbf{j}}\left\{Q \Omega_{2 n-1}\right\},
\end{aligned}
$$

where Eq. (25) is a special case of $n=2$. For pure bosonic case we only take the i-sector in taking the trace. Generalized non-abelian anomaly expression can be obtained in a completely parallel way as in the standard case. It is thus very natural to expect that generalized Chern character $\operatorname{Tr}_{\mathbf{i}}\left\{e^{\mathscr{F}}\right\}$ and $\operatorname{Tr}_{\mathbf{j}}\left\{e^{\mathscr{F}}\right\}$ may play an important role to classify the new "bundle structure" in consideration. From $\Omega_{2 n-1}$ we can construct the physical observables

$$
\begin{aligned}
& O_{\mathrm{e}}^{\mathrm{b}}=\int \operatorname{Tr}_{\mathrm{k}} \Omega_{2 n-1}, \\
& O_{0}^{\mathrm{f}}=\int \operatorname{Tr}_{1} \Omega_{2 n-1},
\end{aligned}
$$

which are invariant under the gauge transformations (11).

We claim that any finite gauge transformation is induced by a gauge element $\mathscr{G}=e^{\mathscr{V}}$ which constitutes mathematically a new "group manifold." A finite gauge transformation of the gauge field is then given by $\mathscr{A} \rightarrow \mathscr{G}^{-1} \mathscr{A} \mathscr{G}+\mathscr{G}^{-1} Q \mathscr{G}$. It is possible to show that the pure gauge term $\mathscr{A} \sim \mathscr{G}^{-1} Q \mathscr{G}$ is a solution of the 
equation of motion (21). Substituting the pure gauge term $\mathscr{A}=\mathscr{G}^{-1} Q^{\mathscr{G}}$ into Eqs. (10), we can construct an action which possesses similar algebraic properties as Wess-Zumino-Witten action [9]

$$
\begin{aligned}
& S_{\mathrm{e}}^{\mathrm{b}}=-\frac{1}{6} \int \operatorname{Tr}_{\mathbf{k}}\left(\mathscr{G}^{-1} Q \mathscr{G}\right)^{3}, \\
& S_{0}^{\mathrm{f}}=-\frac{1}{6} \int \operatorname{Tr}_{\mathbf{1}}\left(\mathscr{G}^{-1} Q \mathscr{G}\right)^{3},
\end{aligned}
$$

which are expected to be topological invariants of this "group manifold."

We have obtained a pure bosonic even-dimensional action which is a special version of more general action which includes gauge fermions and has the same original form as the standard Chern-Simons action. A fermionic odd version of the action emerges as a natural counterpart to the bosonic one. These actions possess a new type of gauge symmetry in the sense that the gauge transformation includes anti-commutators and fermions are included as gauge fields. Series of new gauge invariant observables are proposed. Quaternionic structure is playing a fundamental role in the formulation. We claim that this construction defines mathematically a new "bundle structure" and topological invariants which may be classified by the generalized Chern character.

The algebra of the new gauge symmetry is realized by the Clifford algebra. Explicit analyses of two-dimensional and four-dimensional pure bosonic generalized Chern-Simons actions with the Clifford algebra are given in separate papers $[7,8]$. It turns out that two-dimensional topological gravity appears from the two-dimensional action while we obtain four-dimensional conformal gravity from the four-dimensional version of the generalized Chern-Simons action. It has also been shown in our separate paper [10] that the generalized Chern-Simons actions are equivalent to field theory actions of topological point particle. We believe that our formulation includes new mathematical findings and provides new insights into various physical formulations including four-dimensional gravity.

Acknowledgements. We thank A. Sugamoto and S. Yahikozawa for calling our attention to recent papers. We also thank K. Ueno for useful comments.

\section{References}

1. Chern, S.S.: In: Proc. of 13th Biennial seminar. Canadian Mathematical Congress, pp. 1-40 (1972)

Chern, S.S., Simons, J.: Ann. Math. 99, 48 (1974)

2. Witten, E.: Commun. Math. Phys. 121, 351 (1989)

Moore, G., Seiberg, N.: Phys. Lett. B 220, 422 (1989); Commun. Math. Phys. 123, 177 (1989)

3. Horne, J.H., Witten, E.: Phys. Rev. Lett. 62, 501 (1989)

Witten, E.: Nucl. Phys. B 311, 46 (1988)

4. Witten, E.: Commun. Math. Phys. 117, 353 (1988); Nucl. Phys. B 323, 113 (1989)

5. Horowitz, G.T.: Commun. Math. Phys. 125, 417 (1989)

Myers, R.C., Periwal, V.: Phys. Lett. B 225, 352 (1989)

Karlhede, A., Roček, M.: Phys. Lett. B 224, 58 (1989)

Horowitz, G.T., Srednicki, M.: Commun. Math. Phys. 130, 83 (1990)

Blau, M., Thompson, G.: Ann. Phys. 205, 130 (1991)

6. Marcus, N., Sagnotti, A.: Phys. Lett. B 119, 97 (1982)

7. Kawamoto, N., Watabiki, Y.: Two-dimensional gravity as gauge theory of Clifford algebra from generalized Chern-Simons action. To be published in Phys. Rev. D. Kyoto University preprint, KUNS 1075, HE(TH) 91/07 (1991) 
8. Kawamoto, N., Watabiki, Y.: Four-dimensional conformal gravity from even-dimensional generalized Chern-Simons action. Kyoto University preprint, KUNS 1076, HE(TH) 91/08 (1991)

9. Wess, J., Zumino, B.: Phys. Lett. B 37, 95 (1971)

Witten, E.: Nucl. Phys. B 223, 422 (1983)

10. Kawamoto, N., Watabiki, Y.: Topological particle field theory, general coordinate invariance and generalized Chern-Simons actions. Kyoto University preprint, KUNS 1058, HE(TH) 91/02 (1991)

Communicated by H. Araki 